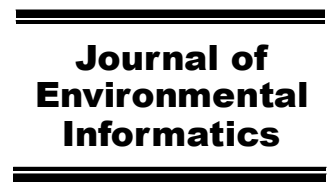

www.iseis.org/jei

\title{
TISEM: A Two-Stage Interval-Stochastic Evacuation Management Model
}

\author{
G. C. $\mathrm{Li}^{1}$, G. H. Huang ${ }^{1, *}$, C. Z. Wu ${ }^{2}$, Y. P. $\mathrm{Li}^{3}$, Y. M. Chen ${ }^{1}$, and Q. Tan ${ }^{1}$ \\ ${ }^{I}$ Faculty of Engineering, University of Regina, Regina, Saskathewan S4S 0A2, Canada \\ ${ }^{2}$ Intelligent Transportation System Research Center, Wuhan University of Technology, Wuhan 430070, P. R. China \\ ${ }^{3}$ College of Urban and Environmental Sciences, Peking University, Beijing 100871, P. R. China
}

Received 8 January 2008; revised 27 May 2008; accepted 28 July 2008; published online 2 September 2008

\begin{abstract}
Traffic allocation planning is commonly required for mass evacuation management. It primarily relies on efficient coordination and appropriate utilization of roadway capacity and available traffic resources. However, traffic and evacuee information are usually difficult to be obtained and consequently of various uncertainties in data. Especially, stochastic information may often exist in evacuation management systems. In this study, a two-stage interval-stochastic evacuation management (TISEM) model was developed for supporting the evacuation planning under uncertainty, by which stochastic and interval evacuation information could be well reflected and communicated in the system. In addition, by adopting the proposed model, a case study abstracted from the City of Wuhan was introduced and solved through an interactive method. Results indicated that useful solutions for planning evacuation routes could be generated based on results of the model. As well, through the model, complex relationships between evacuation time, environmental influences and economic factors could be systematically analyzed. It demonstrated that the proposed TISP model is practical and applicable in real world, and is helpful for authorities to make decisions allocating vehicles before evacuation starts.
\end{abstract}

Keywords: allocation planning, management, optimization, stadium evacuation, transportation, uncertainty

\section{Introduction}

Mass evacuation is required when large-scale assembly events occur, such as those associated with various political, social, economic and sporting activities (Decker et al., 2006; Frantzeskakis and Frantzeskakis, 2006). Such events are normally transient, dynamic and inflexible in their progresses and may lead to intensive evacuation within a short period of time (Hobeika and Kim, 1998; Cova and Johnson, 2003). Therefore, time is usually considered top priority during evacuation processes (Urbanik, 2000; Yi and Zdamar, 2007). At the same time, a series of issues such as economic costs, contaminant emissions and traffic interferences should also be taken into consideration when the evacuation time is to be minimized (Graata et al., 1999; Wu et al., 2008; Tan et al., 2008). Thus, systems analysis techniques could be employed to assist in developing evacuation management plans, which may be helpful for making tradeoffs between meeting required environmental objectives and minimizing system cost.

Previously, many studies were conducted in evacuation management. For example, regional evacuation modeling systems were developed to estimate the evacuation time and the

\footnotetext{
* Corresponding author. Tel.: +1 306 5854095; fax: +1 3065854855. E-mail address: gordon.huang@uregina.ca (G. H. Huang).
}

ISSN: 1726-2135 print/1684-8799 online (C) 2008 ISEIS All rights reserved. doi:10.3808/jei.200800125 traffic flow in road networks (Suleyman, 1995; Bakuli and Smith, 1996). An dynamic traffic assignment model was proposed for supporting evacuation management (Sattayhatewa and Ran, 2000). Cova and Johnson (2003) proposed a network flow model to identify and generate optimal lane-based evacuation routing plans within a complex road network. Yi and Zdamar (2007) proposed an integrated location-routing model for coordinating evacuation operations in response to emergencies and natural disasters. Efforts were also made in reducing environmental effects from evacuations (Grivas and Chaloulakou, 2006). However, in evacuation management, uncertaintyes may exist in the related costs, impact factors and objectives, which will affect the optimization processes and the decision schemes generated (Ettema and Timmermans, 2006). Such complexities and uncertainties have placed the related problems beyond the conventional optimization methods.

Consequently, inexact optimization techniques based on interval-parameter programming (IPP), fuzzy mathematical programming (FMP), and chance-constrained programming (CCP) were employed for evacuation management (Wu et al., 2008; Tan et al., 2008). For example, Waller et al. (2001) and Ettema et al. (2006) systematically analyzed the negative effects of uncertainties during evacuation. In addition, Waller and Ziliaskopoulos (2006) proposed a chance-constrained traffic assignment model in dealing with random time-dependent demand variables with known probability distributions. Furthermore, an inexact optimization model was developed for supporting evacuation management under uncertainty ( $\mathrm{Wu}$ et al., 
2008), through which interval information was successfully introduced into the evacuation modeling systems. Tan et al. (2008) developed an inexact fuzzy robust programming model, through which factors of fuzzy-boundary interval format were successfully introduced as uncertain inputs. Although CCP and FMP can effectively reflect probabilistic and possibilistic uncertainties in a linear model's right-hand sides, they can hardly handle independent uncertainties of the model's left-hand sides and cost coefficients; moreover, none of the FMP, CCP and IPP is capable of examining consequences of violating overriding policies that are considered out of the scope of the planning exercise.

An attractive technique that could help tackle the above shortcomings is the two-stage stochastic programming (TSP). TSP is effective for problems where an analysis of policy scenarios is desired and the related data are mostly uncertain. In TSP, a decision is firstly undertaken before values of random variables are known and, then, after the random events have occurred and their values are known, a second-stage decision is made in order to minimize "penalties" that may appear due to any infeasibility (Loucks et al., 1981; Birge and Louveaux, 1988, 1997; Li et al., 2006). Therefore, the objective of this study is to develop a two-stage interval-stochastic evacuation management (TISEM) model for route planning and vehicle allocation in an evacuation system. The developed model will be able to handle uncertainties expressed as not only probability distributions but also interval values. It can be used for analyzing various policy scenarios that are associated with different levels of penalties when the promised policy targets are violated. This objective entails (a) integrated consideration of evacuation time, transportation costs, and environmental emissions in an evacuation system, (b) incorporation of uncertainties existing as interval values and probability distributions into the modeling formulation, (c) development of an efficient algorithm for solving the T-ISEM model, and (d) application of the T-ISEM model to an case study abstracted from the City of Wuhan.

\section{Modeling Formulation}

Commonly, after a large social event happens, audiences should be transported to several multiple locations, during which time saving, cost reduction and environmental protection are major challenges. Undertaking these challenges primarily relies on efficient coordination and appropriate utilization of roadway capacity and available traffic resources. However, transportation infrastructure is usually a limited resource in terms of accessibility and capacity. Different paths between the event place and multiple destinations may exist. Each path may consist of multiple links. During the evacuation, multiple types of vehicles that have different passenger-loading capacities, fuel consumptions and exhaust emission factors should be allocated to these paths (Wu et al, 2008; Tan et al., 2008). Consequently, varied operational costs, exhaust emissions, and evacuation time may be presented. Therefore, an effective routing plan is desired for making optimal use of the infrastructure.

\subsection{Two-stage Evacuation Management (TSEM) Model}

Stochastic information may exist in evacuation management systems. For example, during an evacuation process, some people may walk away themselves; other people may take public vehicles. Therefore, the number of people who need to be evacuated and that of the related vehicles can hardly be obtained precisely. However, according to the ticket selling and historical data in similar events, the number of passengers may be estimated. Thus, after the original evacuation plan is predefined, a recourse process is required to make modifications. In order to reach the minimum time taken under acceptable economical and environmental considerations, all related factors should considered. Therefore, the problem can be formulated as a TSP model, with minimum time used as the objective function, and the transportation cost and environmental emission as main constraints. The model can be presented as follows:

$$
\begin{aligned}
\operatorname{Min} t & =\sum_{s=1}^{S} \sum_{a=1}^{A_{s}} \sum_{i=1}^{I} x_{s a i} r_{i} \sum_{m \in a} t_{m}+E_{i}\left(\sum_{s=1}^{S} \sum_{a=1}^{A_{s}} \sum_{i=1}^{I} D_{s a i} r_{i} \sum_{m \in a} t_{m}\right) \\
& +\sum_{s=1}^{S} \sum_{a=1}^{A_{s}} \sum_{i=1}^{I} x_{s a i} r_{i} \sum_{k \in a}^{K_{s a}} L t_{k}+E_{i}\left(\sum_{s=1}^{S} \sum_{a=1}^{A_{s}} \sum_{i=1}^{I} D_{s a i} r_{i} \sum_{k \in a}^{K_{s a}} L t_{k}\right) \\
& +\sum_{s=1}^{S} \sum_{a=1}^{A_{s}} \sum_{i=1}^{I} x_{s a i} r_{i} \sum_{b \in a}^{B} T t_{b}+E_{i}\left(\sum_{s=1}^{S} \sum_{a=1}^{A_{s}} \sum_{i=1}^{I} D_{s a i} r_{i} \sum_{b \in a}^{B} T t_{b}\right) \\
& +E_{i}\left(\sum_{s=1}^{S} \sum_{a=1}^{A_{s}} \sum_{i=1}^{I} D_{s a i} r_{i} T e_{i}\right)
\end{aligned}
$$

subject to

$\sum_{a=1}^{A_{s}} \sum_{i=1}^{I}\left(x_{s a i}+D_{s a i}\right) r_{i} \geq R_{s j} \forall s, j$

[passenger capacity constraints]

$\sum_{s}^{S} \sum_{a}^{A_{s}}\left(x_{s a i}+D_{s a i}\right) \leq N_{i} \forall i$

[vehicle quantity constraints]

$$
\begin{aligned}
& \sum_{s=1}^{S} \sum_{a=1}^{A_{s}} \sum_{i=1}^{I}\left(x_{s a i}+D_{s a i}\right) r_{i} G_{s i}-\sum_{s=1}^{S} \sum_{a=1}^{A_{s}} \sum_{i=1}^{I}\left(x_{s a i}+D_{s a i}\right)\left(\sum_{m \in a} l_{m}\right) F_{i} \\
& -\sum_{s=1}^{S} \sum_{a=1}^{A_{s}} \sum_{i=1}^{I} \sum_{m \in a}\left(x_{s a i}+D_{s a i}\right) C_{m i}-\sum_{s=1}^{S} \sum_{a=1}^{A_{s}} \sum_{i=1}^{I} D_{s a i} \cdot C e_{i} \\
& \geq \sum_{s=1}^{S} \sum_{a=1}^{A_{s}} \sum_{i=1}^{I}\left(x_{s a i}+D_{s a i}\right) B e_{i} \\
& \text { [profit constraint] }
\end{aligned}
$$$$
\sum_{s=1}^{S} \sum_{a=1}^{A_{s}} \sum_{i=1}^{I}\left(x_{s a i}+D_{s a i}\right) \sum_{m \in a} l_{m} E C_{m i}+\sum_{s=1}^{S} \sum_{a=1}^{A_{s}} \sum_{i=1}^{I}\left(x_{s a i}+D_{s a i}\right)\left(\sum_{k \in a}^{K} L t_{k}\right) E I C_{i}
$$$$
+\sum_{s=1}^{S} \sum_{a=1}^{A_{s}} \sum_{i=1}^{I}\left(x_{s a i}+D_{s a i}\right)\left(\sum_{b \in a}^{B} T t_{b}\right) E I C_{i} \leq E p
$$

[emission constraint] 
$\sum_{m \in a}^{A_{s}} \sum_{s=1}^{S} \sum_{i=1}^{I}\left(x_{a s i}+D_{a s i}\right)+M X_{m} \leq V M_{m} \forall m$

[traffic constraints]

$x_{s a i}+D_{s a i} \geq 0 \forall s, a, i$

[non-negativity constraints]

$x_{s a i}$ and $D_{s a i}$ are integer, $\forall s, a, i$

[integer constraints]

where:

$A_{s}=$ number of paths from evacuation spot to destination $s$, where $A_{1}=2, A_{2}=3, A_{3}=2$;

$B=$ number of toll stations;

$B e_{i}=$ the lowest profit of type $i$ vehicle (dollar);

$C e_{i}=$ excessive cost assigning one extra type $i$ vehicle (dol-

lar);

$C_{m i}=$ toll of type $i$ vehicle on link $m$ (dollar);

$D_{\text {sai }}=$ deficient type $i$ vehicles to destination $s$ through path $a$, (second-stage decision variable);

$E C_{m i}=\mathrm{CO}$ emission factor of type $i$ vehicle on link $m(\mathrm{~g} / \mathrm{veh} \cdot \mathrm{km})$, $E C_{m i}=\alpha_{i}+\beta_{i} V_{m}+\gamma_{i} V_{m}^{2}$

$E I C_{i}=\mathrm{CO}$ idling emission factor of type $i$ vehicle $(\mathrm{g} / \mathrm{veh} \cdot \mathrm{h})$;

$E p=$ environmental capacity $(\mathrm{CO})$ available in the region

$(\mathrm{g})$

$F_{i}=$ average cost per kilometer of type $i$ vehicle (dollar/ $\mathrm{km})$;

$G_{s}=$ ticket price from evacuation place to destination $s$ (dollar);

$I \quad$ number of vehicle types;

$K=$ number of nodes with traffic light;

$l_{m} \quad=$ length of link $m(\mathrm{~km})$;

$L t_{k}=$ average time delay at node $k$ with traffic light $(\mathrm{h})$;

$m$ = number of links;

$M X_{m}=$ traffic flow on link $m$ (veh);

$N_{i} \quad=$ the maximum number of type $i$ vehicles available;

$R_{s} \quad=$ number of people whose destination is $s$;

$r_{i} \quad=$ full load number of passengers in type $i$ vehicle;

$S \quad$ number of destinations;

$t_{m}=$ travel time on link $m(\mathrm{~h}), t_{m}=l_{m} / v_{m}$;

$T t_{r}=$ average time delay at toll station $k(\mathrm{~h}) ;$

$T e .=$ excessive time taken assigning a type $i$ vehicle;

$v_{m}=$ traffic velocity on link $m(\mathrm{~km} / \mathrm{h})$;

$V M_{m}=$ the maximum traffic capacity on link $m$ (veh);

$x_{\text {sai }}=$ number of type $i$ vehicles whose destination is $s$ on path $a$ (veh), (first-stage decision variable);

$\alpha_{i}, \beta_{i}, \gamma_{i}=$ coefficients of emissions factor for type $i$ vehicles.

Traffic information within a specific link (such as lengths, capacities, traffic flows, and average speeds) can be obtained through various technologies. Some traffic information can al- so be predicted according to real-time historical records. Thus, travel time, transportation profits, and mobile emissions can be obtained. Generally, travel time is the total time used for all passengers to reach their corresponding destinations, which may include running time and traffic delays (due to traffic light and toll-fee payment), as well as extra time that is required in assigning additional vehicles (Southworth, 1991). The profit of transportation companies is the difference of the revenue from ticket sales and the total of operational costs and vehicle tolls. The operational cost includes fuel consumption, vehicles depreciation, drivers' wage, and road-maintenance tax. It is typically dependent on route conditions and vehicle types. Thus, an average operational cost (per kilometer) is used in this study. The environmental emissions, mainly include two parts: emissions when vehicles are running, those when vehicles are in idle status. The first part of emissions is correlated to vehicle number, link length and emission factor; the second part is mainly related to vehicle number, traffic light waiting time and idle emission factor. Generally, emission is a function of speed within a certain link. It can be calculated based on emission factors $\left(E C_{m i}, E I C_{i}\right)$ of each type of vehicles. Herein the emission of $\mathrm{CO}$ is adopted to express the emission levels of vehicles.

To solve the above problem, the distribution of each $R_{s}$ must be converted to an equivalent set of discrete values. Let each $R_{s}$ take values $R_{s j}$ with probabilities $p_{s j}$ for $j=1,2, \ldots, q$, where $j$ defines $q$ levels of the number of people who want to go to destination $s$. Thus, according to Maqsood and Huang (2003, 2004) and Li and Huang (2006), model (1) can be reformulated as follows:

$$
\begin{aligned}
\operatorname{Min} t & =\sum_{s=1}^{S} \sum_{a=1}^{A_{s}} \sum_{i=1}^{I} x_{s a i} r_{i} \sum_{m \in a} t_{m}+\sum_{s=1}^{S} \sum_{a=1}^{A_{s}} \sum_{i=1}^{I} \sum_{j=1}^{q} p_{s j} D_{s a i j} r_{i} \sum_{m \in a} t_{m} \\
& +\sum_{s=1}^{S} \sum_{a=1}^{A_{s}} \sum_{i=1}^{I} x_{s a i} r_{i} \sum_{k \in a}^{K_{s a}} L t_{k}+\sum_{s=1}^{S} \sum_{a=1}^{A_{s}} \sum_{i=1}^{I} \sum_{j=1}^{q} p_{s j} D_{s a i j} r_{i} \sum_{k \in a}^{K_{s a}} L t_{k} \\
& +\sum_{s=1}^{S} \sum_{a=1}^{A_{s}} \sum_{i=1}^{I} x_{s a i} r_{i} \sum_{b \in a}^{B} T t_{b}+\sum_{s=1}^{S} \sum_{a=1}^{A_{s}} \sum_{i=1}^{I} \sum_{j=1}^{q} p_{s j} D_{s a i j} r_{i} \sum_{b \in a}^{B} T t_{b} \\
& +\sum_{s=1}^{S} \sum_{a=1}^{A_{s}} \sum_{i=1}^{I} \sum_{j=1}^{q} p_{s j} D_{s a i j} r_{i} T e_{i}
\end{aligned}
$$

subject to:

$$
\begin{aligned}
& \sum_{a=1}^{A_{s}} \sum_{i=1}^{I}\left(x_{s a i}+D_{s a i j}\right) r_{i} \geq R_{s j} \forall s, j \\
& \sum_{s}^{S} \sum_{a}^{A_{s}}\left(x_{s a i}+D_{s a i j}\right) \leq N_{i} \forall i \\
& \sum_{s=1}^{S} \sum_{a=1}^{A_{s}} \sum_{i=1}^{I}\left(x_{s a i}+D_{s a i j}\right) r_{i} G_{s i}-\sum_{s=1}^{S} \sum_{a=1}^{A_{s}} \sum_{i=1}^{I}\left(x_{s a i}+D_{s a i j}\right)\left(\sum_{m \in a} l_{m}\right) F_{i} \\
& -\sum_{s=1}^{S} \sum_{a=1}^{A_{s}} \sum_{i=1}^{I} \sum_{m \in a}\left(x_{s a i}+D_{s a i j}\right) C_{m i}-\sum_{s=1}^{S} \sum_{a=1}^{A_{s}} \sum_{i=1}^{I} D_{s a i j} \cdot C e_{i} \\
& \geq \sum_{s=1}^{S} \sum_{a=1}^{A_{s}} \sum_{i=1}^{I}\left(x_{s a i}+D_{s a i j}\right) B e_{i} \forall j
\end{aligned}
$$


$\sum_{s=1}^{S} \sum_{a=1}^{A_{s}} \sum_{i=1}^{I}\left(x_{s a i}+D_{s a i j}\right) \sum_{m \in a} l_{m} E C_{m i}+\sum_{s=1}^{S} \sum_{a=1}^{A_{s}} \sum_{i=1}^{I}\left(x_{s a i}+D_{s a i j}\right)\left(\sum_{k \in a}^{K} L t_{k}\right) E I C_{i}$
$+\sum_{s=1}^{S} \sum_{a=1}^{A_{s}} \sum_{i=1}^{I}\left(x_{s a i}+D_{s a i j}\right)\left(\sum_{b \in a}^{B} T t_{b}\right) E I C_{i} \leq E p \forall j$

$\sum_{m \in a}^{A_{s}} \sum_{s=1}^{S} \sum_{i=1}^{I}\left(x_{a s i}+D_{a s i j}\right)+M X_{m} \leq V M_{m} \forall m, j$

$x_{s a i}+D_{s a i j} \geq 0 \forall s, a, i, j$

$x_{\text {sai }}, D_{\text {sai }}$ is integer, $\forall s, a, i, j$

where $D_{\text {saij }}$ is the number of vehicles to be further assigned when the number of passengers to destination $s$ is $R_{s j}$ with probability $p_{s j}$ and the existing number of type $i$ vehicles $\left(x_{s a i}\right)$ are not insufficient in the given routes.

\subsection{Two-stage Interval-Stochastic Evacuation Manage- ment (TISEM) Model}

As described above, uncertainties in the number of passengers who are going to given destinations can be reflected in model (2) as probability density functions. As well, randomness in other factors (e.g., number of passengers in each type of vehicles, average speed and traffic flow of every link, waiting time, operational cost, and emission of each type of vehicles) also needs to be addressed. However, in evacuation management systems, the quality of uncertain information may not be satisfactory enough to be expressed as probability distributions; even if the probability distributions are available, it could be difficult to reflect them in large-scale models. Consequently, interval parameters are introduced into the stochastic programming framework to facilitate communication of the uncertainties into the optimization process, resulting in a TISEM model as follows:

$$
\begin{aligned}
\operatorname{Min} t^{ \pm} & =\sum_{s=1}^{S} \sum_{a=1}^{A_{s}} \sum_{i=1}^{I} x_{s a i}^{ \pm} r_{i}^{ \pm} \sum_{m \in a} t_{m}^{ \pm}+\sum_{s=1}^{S} \sum_{a=1}^{A_{s}} \sum_{i=1}^{I} \sum_{j=1}^{q} p_{s j} D_{s a j i}^{ \pm} r_{i}^{ \pm} \sum_{m \in a} t_{m}^{ \pm} \\
& +\sum_{s=1}^{S} \sum_{a=1}^{A_{s}} \sum_{i=1}^{I} x_{s a i}^{ \pm} \sum_{i}^{ \pm} \sum_{k \in a}^{K_{s a}} L t_{k}^{ \pm}+\sum_{s=1}^{S} \sum_{a=1}^{A_{s}} \sum_{i=1}^{I} \sum_{j=1}^{q} p_{s j} D_{s a i j}^{ \pm} r_{i}^{ \pm} \sum_{k \in a}^{K_{s a}} L t_{k}^{ \pm} \\
& +\sum_{s=1}^{S} \sum_{a=1}^{A_{s}} \sum_{i=1}^{I} x_{s a i}^{ \pm} \Gamma_{i}^{ \pm} \sum_{b \in a}^{B} T t_{b}^{ \pm}+\sum_{s=1}^{S} \sum_{a=1}^{A_{s}} \sum_{i=1}^{I} \sum_{j=1}^{q} p_{s j} D_{s a i j}^{ \pm} r_{i}^{ \pm} \sum_{b \in a}^{B} T t_{b}^{ \pm} \\
& +\sum_{s=1}^{S} \sum_{a=1}^{A_{s}} \sum_{i=1}^{I} \sum_{j=1}^{q} p_{s j} D_{s a j}^{ \pm} r_{i}^{ \pm} T e_{i}^{ \pm}
\end{aligned}
$$

subject to:

$$
\begin{aligned}
& \sum_{a=1}^{A s} \sum_{i=1}^{I}\left(x_{s a i}^{ \pm}+D_{\text {saij }}^{ \pm}\right) r_{i} \geq R_{s j}^{ \pm} \forall s, j \\
& \sum_{s}^{S} \sum_{a}^{A_{s}}\left(x_{\text {sai }}^{ \pm}+D_{\text {saij }}^{ \pm}\right) \leq N_{i} \forall i
\end{aligned}
$$

$$
\begin{aligned}
& \sum_{s=1}^{S} \sum_{a=1}^{A_{s}} \sum_{i=1}^{I}\left(x_{s a i}^{ \pm}+D_{s a i j}^{ \pm}\right) r_{i}^{ \pm} G_{s i}-\sum_{s=1}^{S} \sum_{a=1}^{A_{s}} \sum_{i=1}^{I}\left(x_{s a i}^{ \pm}+D_{s a i j}^{ \pm}\right) F_{i}^{ \pm} \sum_{m \in a} l_{m} \\
& -\sum_{i=1}^{S} \sum_{a=1}^{A_{s}} \sum_{i=1}^{I} \sum_{m \in a}\left(x_{s a i}^{ \pm}+D_{s a i j}^{ \pm}\right) C_{m i}-\sum_{s=1}^{S} \sum_{a=1}^{A_{s}} \sum_{i=1}^{I} D_{s a i j}^{ \pm} C e_{i}^{ \pm} \\
& \geq \sum_{s=1}^{S} \sum_{a=1}^{A_{s}} \sum_{i=1}^{I}\left(x_{s a i}^{ \pm}+D_{s a i j}^{ \pm}\right) B e_{i}^{ \pm} \forall j \\
& \sum_{s=1}^{S} \sum_{a=1}^{A_{s}} \sum_{i=1}^{I}\left(x_{s a i}^{ \pm}+D_{s a i j}^{ \pm}\right) \sum_{m \in a} l_{m} E C_{m i}^{ \pm}+\sum_{s=1}^{S} \sum_{a=1}^{A_{s}} \sum_{i=1}^{I}\left(x_{s a i}^{ \pm}+D_{s a i j}^{ \pm}\right) E I C_{i}^{ \pm} \sum_{k \in a}^{K} L t_{k}^{ \pm} \\
& +\sum_{s=1}^{S} \sum_{a=1}^{A s} \sum_{i=1}^{I}\left(x_{s a i}^{ \pm}+D_{s a i j}^{ \pm}\right)\left(\sum_{b \in a}^{B} T t_{b}^{ \pm}\right) E I C_{i}^{ \pm} \leq E p^{ \pm} \forall h
\end{aligned}
$$

$\sum_{m \in a}^{A_{s}} \sum_{s=1}^{S} \sum_{i=1}^{I}\left(x_{s a i}^{ \pm}+D_{s a i j}^{ \pm}\right)+M X_{m}^{ \pm} \leq V M_{m} \forall m, j$

$x_{s a i}^{ \pm}+D_{s a i j}^{ \pm} \geq 0 \forall s, a, i, j$

$D_{\text {saij }}^{ \pm}$is integer, $\forall s, a, i, j$

According to Huang and Louck (2000). let $x_{s a i}^{ \pm}=x_{s a i}^{-}+$ $\Delta x_{s a i} y_{s a i}$, where $\Delta x_{s a i}=x_{s a i}^{+}-x_{s a i}^{-}$and $y_{s a i} \in[0,1]$. An nptimized set of target values can be obtained by having $y_{\text {sai }}$ in model (3) as decision variables. Then model (3) can be converted to:

$$
\begin{aligned}
& \operatorname{Min} t^{ \pm}=\sum_{s=1}^{3} \sum_{a=1}^{A_{s}} \sum_{i=1}^{3}\left(x_{s a i}^{-}+\Delta x_{s a i} y_{s a i}\right) r_{i}^{ \pm} \sum_{m \in a} t_{m}^{ \pm}+\sum_{s=1}^{3} \sum_{a=1}^{A_{s}} \sum_{i=1}^{3} \sum_{j=1}^{q} p_{s j} D_{s a j}^{ \pm} r_{i}^{ \pm} \sum_{m \in a} t_{m}^{ \pm} \\
& +\sum_{s=1}^{3} \sum_{a=1}^{A_{s}} \sum_{i=1}^{3}\left(x_{s a i}^{-}+\Delta x_{s a i} y_{s a i}\right) r_{i}^{ \pm} \sum_{k \in a}^{K_{s a}} L t_{k}^{ \pm}+\sum_{s=1}^{3} \sum_{a=1}^{A_{s}} \sum_{i=1}^{3} \sum_{j=1}^{q} p_{s j} D_{s a i j}^{ \pm} r_{i}^{ \pm} \sum_{k \in a}^{K_{s a}} L t_{k}^{ \pm} \\
& +\sum_{s=1}^{3} \sum_{a=1}^{A_{s}} \sum_{i=1}^{3}\left(x_{s a i}^{-}+\Delta x_{s a i} y_{s a i}\right) r_{i}^{ \pm} \sum_{b \in a}^{B} T t_{b}^{ \pm}+\sum_{s=1}^{3} \sum_{a=1}^{A_{s}} \sum_{i=1}^{3} \sum_{j=1}^{q} p_{s j} D_{s a j}^{ \pm} r_{i}^{ \pm} \sum_{b \in a}^{B} T t_{b}^{ \pm} \\
& +\sum_{s=1}^{3} \sum_{a=1}^{A_{s}} \sum_{i=1}^{3} \sum_{j=1}^{q} p_{s j} D_{s a i j}^{ \pm} r_{i}^{ \pm} T e_{i}^{ \pm}
\end{aligned}
$$

subject to:

$$
\begin{aligned}
& \sum_{a=1}^{A s} \sum_{i=1}^{3}\left(x_{s a i}^{-}+\Delta x_{s a i} y_{s a i}+D_{s a i j}^{ \pm}\right) r_{i}^{ \pm} \geq R_{s j}^{ \pm} \forall s, j \\
& \sum_{s}^{S} \sum_{a}^{A_{s}}\left(x_{s a i}^{-}+\Delta x_{s a i} y_{s a i}+D_{s a i j}^{ \pm}\right) \leq N_{i} \forall i \\
& \sum_{s=1}^{3} \sum_{a=1}^{A} \sum_{i=1}^{3}\left(x_{s a i}^{-}+\Delta x_{s a i} y_{s a i}+D_{s a i j}^{ \pm}\right) r_{i}^{ \pm} G_{s i}-\sum_{s=1}^{3} \sum_{a=1}^{4} \sum_{i=1}^{4} F_{i}^{ \pm}\left(x_{s a i}^{-}+\Delta x_{s a i} y_{s a i}+D_{s a i j}^{ \pm}\right) \\
& \sum_{m \in a} l_{m}-\sum_{i=1}^{3} \sum_{a=1}^{4} \sum_{i=1}^{3} \sum_{m \in a}\left(x_{s a i}^{-}+\Delta x_{s a i} y_{s a i}+D_{s a i j}^{ \pm}\right) C_{m i}-\sum_{s=1}^{3} \sum_{a=1}^{4} \sum_{i=1}^{3} D_{s a j j}^{ \pm} C e_{i}^{ \pm} \\
& \geq \sum_{s=1}^{3} \sum_{a=1}^{4} \sum_{i=1}^{3}\left(x_{s a i}^{-}+\Delta x_{s a i} y_{s a i}+D_{s a i j}^{ \pm}\right) B e_{i}^{ \pm} \forall j
\end{aligned}
$$


$\sum_{s=1}^{3} \sum_{a=1}^{A_{s}} \sum_{i=1}^{3}\left(x_{s a i}^{-}+\Delta x_{s a i} y_{s a i}+D_{s a i j}^{ \pm}\right) \sum_{m \in a} l_{m} E C_{m i}^{ \pm}$

$+\sum_{s=1}^{3} \sum_{a=1}^{A_{s}} \sum_{i=1}^{3}\left(x_{s a i}^{-}+\Delta x_{s a i} y_{s a i}+D_{s a i j}^{ \pm}\right) E I C_{i}^{ \pm} \sum_{k \in a}^{K} L t_{k}^{ \pm}$

$+\sum_{s=1}^{3} \sum_{a=1}^{A s} \sum_{i=1}^{3}\left(x_{s a i}^{-}+\Delta x_{s a i} y_{s a i}+D_{s a i j}^{ \pm}\right)\left(\sum_{b \in a}^{B} T t_{b}^{ \pm}\right) E I C_{i}^{ \pm} \leq E p^{ \pm} \forall h$

$\sum_{m \in a}^{A_{s}} \sum_{s=1}^{3} \sum_{i=1}^{3}\left(x_{s a i}^{-}+\Delta x_{s a i} y_{s a i}+D_{s a i j}^{ \pm}\right)+M X_{m}^{ \pm} \leq V M_{m} \forall m, j$

$x_{s a i}^{-}+\Delta x_{s a i} y_{s a i}+D_{s a i j}^{ \pm} \geq 0 \forall s, a, i, j$

$0 \leq y_{\text {sai }} \leq 1$

$x_{s a i}^{-}+\Delta x_{s a i} y_{s a i}$ and $D_{s a i j}^{ \pm}$are integer, $\forall s, a, i, j$

where $t^{ \pm}=l / V_{\ldots}^{\mp}: E C_{\ldots:}^{ \pm}=\alpha_{:}+\beta_{:} V_{\ldots}^{ \pm}+\gamma_{i}\left(V_{\ldots}^{ \pm}\right)^{2}: B e_{i}^{ \pm}, C e^{ \pm}$, $E C_{m i}^{ \pm}, E I C_{i}^{ \pm}, E p^{ \pm}, L t_{k}^{ \pm}, M X_{m}^{ \pm}, r_{i}^{ \pm}, t_{m}^{ \pm}, T e_{i}^{ \pm}, T t_{b}^{ \pm}, V_{m}^{\mp}, x_{s a i}^{ \pm}$and $D_{s a i j}^{ \pm}$ are interval parameters and variables. For example, letting $x_{s a i}^{-}$and $x^{+}$be lower and upper bounds of $x_{s a i}^{ \pm}$, respectively, we have $x_{s a i}^{ \pm}=\left[x_{s a i}^{-}, x_{s a i}^{+}\right]$.

\subsection{Solution Method}

Model (4) is a single objective interval-parameter programming model. According to Huang et al. $(1993,1994)$ and Maqsood and Huang (2003), it can be solved through decomposition into two submodels. The submodel corresponding to the lower bound of the objective function value can be formulated as follows:

$$
\begin{aligned}
& \operatorname{Min} t^{-}=\sum_{s=1}^{3} \sum_{a=1}^{A_{s}} \sum_{i=1}^{3}\left(x_{s a i}^{-}+\Delta x_{s a i} y_{s a i}\right) r_{i}^{-} \sum_{m \in a} t_{m}^{-}+\sum_{s=1}^{3} \sum_{a=1}^{A_{s}} \sum_{i=1}^{3} \sum_{j=1}^{q} p_{s j} D_{s a i j}^{-} r_{i}^{-} \sum_{m \in a} t_{m}^{-} \\
& +\sum_{s=1}^{3} \sum_{a=1}^{A_{s}} \sum_{i=1}^{3}\left(x_{s a i}^{-}+\Delta x_{s a i} y_{s a i}\right) r_{i}^{-} \sum_{k \in a}^{K_{s a}} L t_{k}^{-}+\sum_{s=1}^{3} \sum_{a=1}^{A_{s}} \sum_{i=1}^{3} \sum_{j=1}^{q} p_{s j} D_{s a i j}^{-} r_{i}^{-} \sum_{k \in a}^{K_{s a}} L t_{k}^{-} \\
& +\sum_{s=1}^{3} \sum_{a=1}^{A_{s}} \sum_{i=1}^{3}\left(x_{s a i}^{-}+\Delta x_{s a i} y_{s a i}\right) r_{i}^{-} \sum_{b \in a}^{B} T t_{b}^{-}+\sum_{s=1}^{3} \sum_{a=1}^{A_{s}} \sum_{i=1}^{3} \sum_{j=1}^{q} p_{s j} D_{s a i j}^{-} r_{i}^{-} \sum_{b \in a}^{B} T t_{b}^{-} \\
& +\sum_{s=1}^{3} \sum_{a=1}^{A_{s}} \sum_{i=1}^{3} \sum_{j=1}^{q} p_{s j} D_{s a i j}^{-} r_{i}^{-} T e_{i}^{-}
\end{aligned}
$$

subject to:

$$
\begin{aligned}
& -\sum_{a=1}^{A s} \sum_{i=1}^{3} r_{i}^{+}\left(x_{s a i}^{-}+\Delta x_{s a i} y_{s a i}+D_{s a i j}^{-}\right) \leq-R_{s j}^{-} \forall j, s \\
& \sum_{s}^{S} \sum_{a}^{A_{s}}\left(x_{s a i}^{-}+\Delta x_{s a i} y_{s a i}+D_{s a i j}^{-}\right) \leq N_{i} \forall i
\end{aligned}
$$

$\sum_{s=1}^{3} \sum_{a=1}^{A_{s}} \sum_{i=1}^{3}\left(x_{s a i}^{-}+\Delta x_{s a i} y_{s a i}\right)\left\{\left(\sum_{m \in a} l_{m}\right) F_{i}^{-}+\sum_{m \in a} C_{m i}+B e_{i}^{-}-r_{i}^{+} G_{s i}\right\}$

$+\sum_{s=1}^{3} \sum_{a=1}^{A_{s}} \sum_{i=1}^{3} D_{s a i j}^{-}\left\{\left(\sum_{m \in a} l_{m}\right) F_{i}^{-}+\sum_{m \in a} C_{m i}+B e_{i}^{-}+C e_{i}^{-}-r_{i}^{+} G_{s i}\right\} \leq 0 \forall j$

$$
\begin{aligned}
& \sum_{s=1}^{3} \sum_{a=1}^{A_{s}} \sum_{i=1}^{3}\left(x_{s a i}^{-}+{ }_{\Delta} x_{s a i} y_{s a i}\right)\left\{\sum_{m \in a} l_{m} E C_{m i}^{+}+\left(\sum_{k \in a}^{K} L t_{k}^{+}\right) E I C_{i}^{+}+\left(\sum_{b \in a}^{B} T t_{b}^{+}\right) E I C_{i}^{+}\right\} \\
& +\sum_{s=1}^{3} \sum_{a=1}^{A_{s}} \sum_{i=1}^{3} D_{s a i j}^{-}\left\{\sum_{m \in a} l_{m} E C_{m i}^{+}+\left(\sum_{k \in a}^{K} L t_{k}^{+}\right) E I C_{i}^{+}+\left(\sum_{b \in a}^{B} T t_{b}^{+}\right) E I C_{i}^{+}\right\} \leq E p^{+} \forall j
\end{aligned}
$$

$\sum_{m \in a}^{A_{s}} \sum_{s=1}^{3} \sum_{i=1}^{3}\left(x_{s a i}^{-}+\Delta x_{s a i} y_{s a i}+D_{s a i j}^{-}\right) \leq V M_{m}-M X_{m}^{-} \forall m, j$

$x_{s a i}^{-}+\Delta x_{s a i} y_{s a i}+D_{s a i j}^{-} \geq 0 \forall s, a, i, j$

$0 \leq y_{\text {sai }} \leq 1$

$x_{s a i}^{-}+\Delta x_{s a i} y_{s a i}$ and $D_{s a i j}^{-}$are integer, $\forall s, a, i, j$

where $D_{\text {saii }}^{-}$and $y_{\text {sai }}$ are decision variables. Let $D_{\text {saij opt }}^{-}$ and $y_{\text {sai opt }}$ be solutions of model (5). Then the optimized pre-assigned type $i$ vehicles on path $a$ to destination $s$ are $x_{\text {sai opt }}^{ \pm}=x_{\text {sai }}^{-}+\Delta x_{\text {sai }} y_{\text {sai opt }}$. Thus, according to Huang (1996), the submodel corresponding to the upper bound of the objective function value (i.e. Min $t^{+}$) is:

$$
\begin{aligned}
& \operatorname{Min} t^{+}=\sum_{s=1}^{3} \sum_{a=1}^{A_{s}} \sum_{i=1}^{3}\left(x_{\text {sai }}^{-}+\Delta x_{\text {sai }} y_{\text {sai opt }}\right) r_{i}^{+} \sum_{m \in a} t_{m}^{+}+\sum_{s=1}^{3} \sum_{a=1}^{A_{s}} \sum_{i=1}^{3} \sum_{j=1}^{q} p_{s j} D_{\text {saij }}^{+} r_{i}^{+} \sum_{m \in a} t_{m}^{+} \\
& +\sum_{s=1}^{3} \sum_{a=1}^{A_{s}} \sum_{i=1}^{3}\left(x_{\text {sai }}^{-}+\Delta x_{\text {sai }} y_{\text {sai opt }}\right) r_{i}^{+} \sum_{k \in a}^{K_{s a}} L t_{k}^{+}+\sum_{s=1}^{3} \sum_{a=1}^{A_{s}} \sum_{i=1}^{3} \sum_{j=1}^{q} p_{s j} D_{s a i j}^{+} r_{i}^{+} \sum_{k \in a}^{K_{s a}} L t_{k}^{+} \\
& \quad+\sum_{s=1}^{3} \sum_{a=1}^{A_{s}} \sum_{i=1}^{3}\left(x_{s a i}^{-}+\Delta x_{\text {sai }} y_{\text {sai opt }}\right) r_{i}^{+} \sum_{b \in a}^{B} T t_{b}^{+}+\sum_{s=1}^{3} \sum_{a=1}^{A_{s}} \sum_{i=1}^{3} \sum_{j=1}^{q} p_{s j} D_{\text {saij }}^{+} r_{i}^{+} \sum_{b \in a}^{B} T t_{b}^{+} \\
& \quad+\sum_{s=1}^{3} \sum_{a=1}^{A_{s}} \sum_{i=1}^{3} \sum_{j=1}^{q} p_{s j} D_{\text {saij }}^{+} r_{i}^{+} T e_{i}^{+}
\end{aligned}
$$

subject to:

$$
-\sum_{a=1}^{A s} \sum_{i=1}^{3} r_{i}^{-}\left(x_{s a i}^{-}+\Delta x_{s a i} y_{\text {sai opt }}+D_{s a i j}^{+}\right) \leq-R_{s j}^{+} \forall s, j
$$

$\sum_{s}^{S} \sum_{a}^{A_{s}}\left(x_{s a i}^{-}+\Delta x_{\text {sai }} y_{\text {sai opt }}+D_{\text {saij }}^{+}\right) \leq N_{i} \forall i$

$$
\begin{aligned}
& \sum_{s=1}^{3} \sum_{a=1}^{A_{s}} \sum_{i=1}^{3}\left(x_{s a i}^{-}+\Delta x_{\text {sai }} y_{\text {sai opt }}\right)\left\{\left(\sum_{m \in a} l_{m}\right) F_{i}^{+}+\sum_{m \in a} C_{m i}+B e_{i}^{+}-r_{i}^{-} G_{s i}\right\} \\
& +\sum_{s=1}^{3} \sum_{a=1}^{A_{s}} \sum_{i=1}^{3} D_{s a i j}^{+}\left\{\left(\sum_{m \in a} l_{m}\right) F_{i}^{+}+\sum_{m \in a} C_{m i}+B e_{i}^{+}+C e_{i}^{+}-r_{i}^{-} G_{s i}\right\} \leq 0 \forall j
\end{aligned}
$$

$$
\begin{aligned}
& \sum_{s=1}^{3} \sum_{a=1}^{A_{s}} \sum_{i=1}^{3}\left(x_{s a i}^{-}+\Delta x_{\text {sai }} y_{\text {sai opt }}\right)\left(\sum_{m \in a} l_{m} E C_{m i}^{-}+E I C_{i}^{-} \cdot \sum_{k \in a}^{K} L t_{k}^{-}+E I C_{i}^{-} \cdot \sum_{b \in a}^{B} T t_{b}^{+}\right) \\
& +\sum_{s=1}^{3} \sum_{a=1}^{A_{s}} \sum_{i=1}^{3} D_{s a i j}^{+}\left(\sum_{m \in a} l_{m} E C_{m i}^{-}+E I C_{i}^{-} \cdot \sum_{k \in a}^{K} L t_{k}^{-}+E I C_{i}^{-} \cdot \sum_{b \in a}^{B} T t_{b}^{-}\right) \leq E p^{-} \forall j
\end{aligned}
$$


$\sum_{m \in a}^{A_{s}} \sum_{s=1}^{3} \sum_{i=1}^{3}\left(x_{s a i}^{-}+\Delta x_{\text {sai }} y_{\text {sai opt }}+D_{\text {saij }}^{+}\right) \leq V M_{m}-M X_{m}^{+} \forall m, j$

$x_{\text {sai }}^{-}+\Delta x_{\text {sai }} y_{\text {sai opt }}+D_{\text {saij }}^{-} \geq 0 \forall s, a, i, j$

$D_{\text {saij }}^{+} \geq D_{\text {saij opt }}^{-} \forall s, a, i, j$

$D_{\text {saij }}^{+}$is integer, $\forall s, a, i, j$

where $D_{\text {saij }}^{+}$are decision variables. Sub-models (5) and (6) are deterministic LP problems. Thus, according to Huang et al. (1993), solutions for model (4) under the optimized allowable-vehicle levels are:

$t_{\text {opt }}^{ \pm}=\left[t^{-}{ }_{\text {opt }}, t^{+}{ }_{\text {opt }}\right] ; D_{\text {saij opt }}^{ \pm}=\left[D_{\text {saij opt }}^{-}, D_{\text {saij opt }}^{+}\right] \forall s, a, i, j ;$

where $t^{-}$opt and $D^{-}$opt are from solution of sub-model (5), and ${t^{+}}_{\text {opt }}$ and $D_{\text {saij opt }}^{+}$are from that of sub-model (6). Thus, the optimum assignment of the vehicles is:

$A_{\text {sai opt }}^{ \pm}=x_{\text {sai opt }}^{ \pm}+\mathrm{D}_{\text {saij opt }}^{ \pm} \quad \forall s, a, i, j$

Figure 1 shows the schematic of the modeling methodology. In the following, solution algorithm of the TISP model with the objective being minimized is presented in a pseudocode format as follows:

Step 1. Formulate TISP model (3).

Step 2. Reformulate the TISP model by introducing $x_{s a i}^{ \pm}=$ $x_{s a i}^{-}+\Delta x_{s a i} y_{s a i}$, where $x_{s a i}=x_{s a i}^{+}-x_{s a i}^{-}$and $y_{s a i} \in[0,1]$; this leads to model (4).

Step 3. Transform the TISP model [i.e. model (4)] into two sub-models, where the lower bound of the $t^{ \pm}$is desired since the objective is to minimize $t^{ \pm}$.

Step 4. Formulate Min $t^{-}$sub-model (5).

Step 5. Solve the Min $t^{-}$sub-model, and obtain $D_{\text {saij opt }}^{-}$and $y_{\text {sai opt }}$.

Step 6. Calculate $x_{\text {sai opt }}^{ \pm}=x_{\text {sai }}^{-}+\Delta x_{\text {sai }} y_{\text {sai opt }}$.

Step 7. Calculate $t_{o p t}^{-}$.

Step 8. Formulate $\mathrm{Min} t^{+}$sub-model (6).

Step 9. Solve the $\operatorname{Min} t^{+}$sub-model, and obtain $D_{\text {saij opt }}^{+}$.

Step 10. Calculate $t_{o p t}^{+}$.

Step 11. Solutions of the TISP model are: $t_{\text {opt }}^{ \pm}=\left[t_{\text {opt }}^{-}, t_{\text {opt }}^{+}\right]$and $D_{\text {saij opt }}^{ \pm}=\left[D_{\text {saij opt }}^{-}, D_{\text {saij opt }}^{+}\right] \forall s, a, i, j$.

Step 12. Thus, we have the optimized assignment of vehicles: $A_{\text {saij opt }}^{ \pm}=x_{\text {sai opt }}^{ \pm}+D_{\text {saij opt }}^{ \pm} \quad \forall s, a, i, j$.

Step 13. Stop.

\section{Case Study}

The study system is a part of the traffic network within the City of Wuhan, which is the capital of Hubei Province, China. The city is recognized as the political, economic, cultural, and transportation center of central China. Passenger transportations within the city mainly rely on vehicles. With the development of economy and the consequent increase of traffic vehicles, the City of Wuhan is suffering from the increasing emissions of air pollutants from transportation sources ( $\mathrm{Lv}$ et al., 2006; Querol et al., 2006). Such problems are presented to be intensified especially during evacuations of mega-events (such as sports games). As the biggest stadium of the city, the Wuhan Sports Center Stadium (WSCS) has a capacity for containing 60,000 people. It can also provide space for parking of 3000 cars and 300 buses at the same time. In 2007, the FIFA Women's World Cup and the $6^{\text {th }}$ Intercity Games were successfully held there. However, some problems were disclosed during the evacuations, such as limited capacity at several road links, consequent delays in the transportation system, and excess emissions of air pollutants.

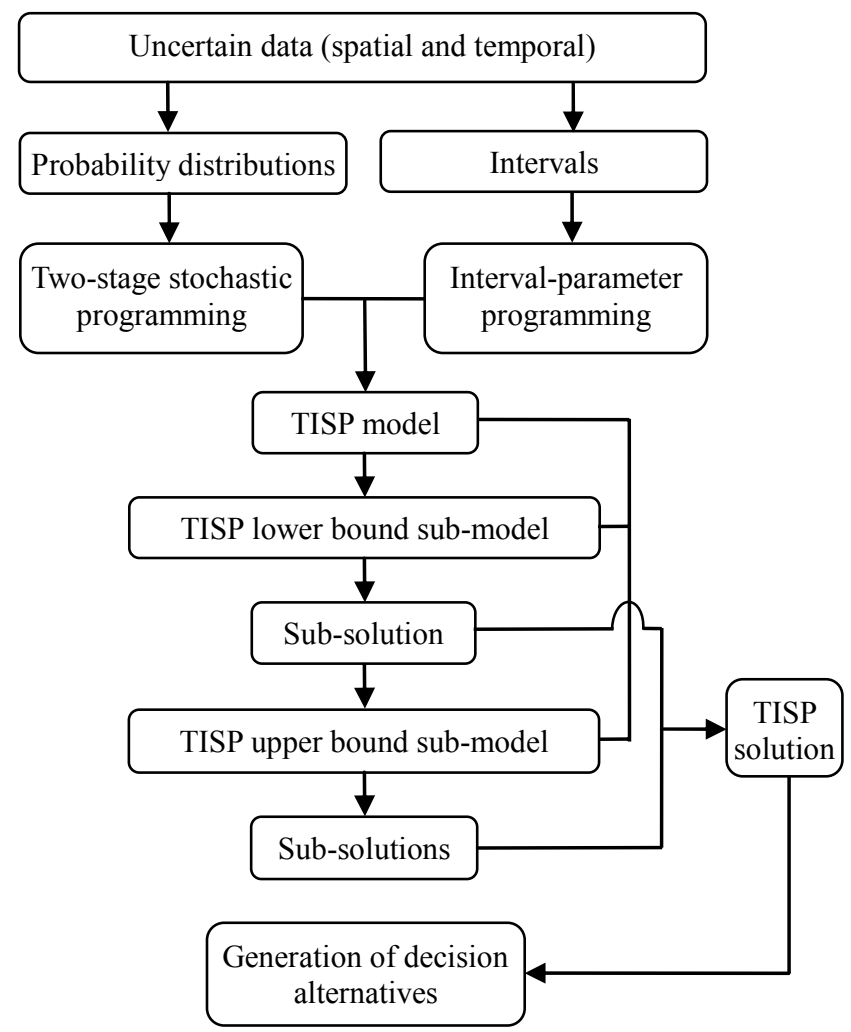

Figure 1. Schematic of the TISP methodology.

To improve evacuations after major events, the developed TISEM can be used for supporting the planning of evacuation management. The upcoming 2009 Wuhan City Games is to be held at the Wuhan Sport Center Stadium in 2009. During the Games, after each competition activity, ten thousands of audiences need to be evacuated. Among them, some audiences (usually of 20 to 30 percent) who live in the urban area may use the public transportation system. However, thousands of the other people ( 70 to 80 percent) still need to commute to 
bus or train stations first, and then to other towns or communities. In this study, one evacuation spot (Wuhan Sports Center Stadium) and three destinations (Qiaokou Bus Station, Hankou Long Distance Bus Station, and Wuchang Railway Station) are considered (Figure 2).

Based on the spatial relationships among the evacuation spot and the destinations, a study network is formulated without loss of generality. This network includes eight links, two traffic lights and one toll station, as shown in Figure 3. To reach the three destinations, seven alternative paths exist. In detail, there are two (i.e. paths 11 and 12), three (i.e. paths 21 , 22 and 23) and two (i.e. paths 31 and 32) possible paths for destinations 1, 2 and 3, respectively. The lengths $(L)$, the average traffic flow speeds $(V)$, the maximum capacities $(V M)$, and the predicted normal traffic flows $(M X)$ of the links are shown in Table 1. The adopted evacuation vehicles can be classified into three types according to their loading capacities $(r)$, which are associated with different operational costs $(F)$, ticket prices $(G)$, available numbers $(N)$ and emission factors ( $\alpha, \beta, \gamma$ and EIC) (Table 1). Numbers of evacuees to the three destinations and the associated probabilities are given in Table 2. Table 3 presents the vehicle allocation plans (i.e. first-stage decisions) predefined by the local authority, which should be adjusted by assigning extra vehicles to the paths when the random numbers of evacuees are realized to certain values. Extra cost $(\mathrm{Ce})$ and extra time $(\mathrm{Te})$ corresponding to the corrective actions at the second stage are also shown in Table 1. Considering the limitations in traffic resources, the requirements for minimum profits and the targets of pollutant emissions, the concerned problems are: (i) how to effectively allocate the vehicles from the vehicle centers to the paths in order to minimize the total evacuation time under uncertainty, and (ii) how to meet the evacuation demands and fulfill the economic and environmental targets under the least system disruption. Since uncertainties exist in a variety of system components and a linkage to economic consequences is desired, TISEM is considered as an effective approach for tackling such a problem.

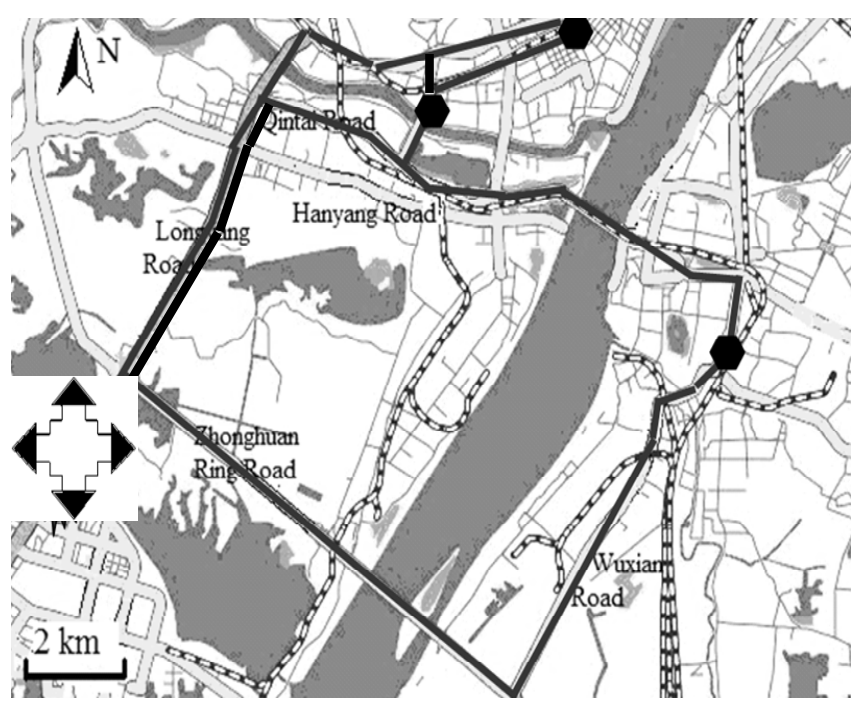

Figure 2. The evacuation routes.
The solutions obtained from the TISEM model are displayed in Table 4. The obtained objective function value (i.e. minimized total time) would be [3556, 6181] hrs. Three sets of solutions for the decision variables are obtained corresponding to the three probability levels of evacuee numbers. Table 4 suggests that, under a low level of evacuee numbers $(j=1)$, a total of $[68,82]$ vehicles would be allocated for evacuating persons to destination 1 , among which only 6 would be arranged to path 11 , while $[62,76]$ would be allocated to path 12 . The reason is that the travel time on path 11 is longer than that on path 12 . For reaching destination 1 , the medium-sized vehicles would be dominant (i.e. 53), followed by large-sized ones (i.e. $[15,29])$. As for destination 2 , the numbers of vehicles allocated to paths 21, 22 and 23 would be 1, 34 and [41, 57], respectively. Paths 22 and 23 would be the main routes for destination 2 due to their relatively shorter distances. In terms of vehicles sizes, [16, 17] small-size, [31, 32] medium-size and $[29,43]$ large-size vehicles would be allocated for destination 2. To reach destination $3,[36,42]$ vehicles would take path 31 , and only 5 would take path 32 . It is indicated that, most of the vehicles allocated to destination 3 are of large size (with only 1 small-size vehicle being included). This is due to the fact that the large-sized vehicles have lower unit transportation costs (dollar per passenger) and $\mathrm{CO}$ emission rates (gram per passenger) compared to vehicles of smaller sizes.

Under a medium level of evacuee numbers $(j=2)$, there would be $[6,27]$ vehicles on path 11 , and $[71,76]$ vehicles on path 12. Among them, the medium-sized vehicles would be in majority $([53,74])$, followed by large-sized ones $([23,27])$ and then small-sized ones $([1,2])$. For destination 2 , the numbers of vehicles allocated to paths 21,22 and 23 would be [1, 2], 34 and [50, 66], respectively. Due to their relatively shorter distances, paths 22 and 23 would still be the main routes to reach destination 2 . The numbers of allocated small-, medium-, and large-sized vehicles would be $[17,18],[31,32]$ and $[37$, $52]$, respectively. For destination $3,[38,66]$ vehicles would be allocated to path 31 , while 5 vehicles would take path 32 . Vehicles of large size would be the dominant ones. Compared with the allocation plans under a lower level of evacuee numbers, more small-sized vehicles would be allocated under this condition. This is probably attributed to the increased evacuation demands associated with the raised numbers of evacuees.

As shown in Table 4, under a high level of evacuee numbers $(j=3)$, the evacuation plan for destination 1 would be similar to those under the low and medium levels of evacuees. In detail, [6, 35] vehicles would take path 11 , while [80, 83] ones would be allocated to path 12 . Most of these vehicles would be of medium size ([53, 84]), and the large- and smallsized vehicles would be 31 and [2, 3], respectively. As for destination 2 , there would be $[1,33],[34,41]$ and 59 vehicles on paths 21,22 and 23, respectively. A total of [17, 18] smallsized, [31, 32] medium-sized and [37, 52] large-sized vehicles would be adopted for evacuating event-participants to destination 2. It appears that small-sized vehicles would be more likely to be employed on path 21 which has a longer distance. This is because large-sized vehicles are competitive in both transportation costs and $\mathrm{CO}$ emission ratios, which would thus be 


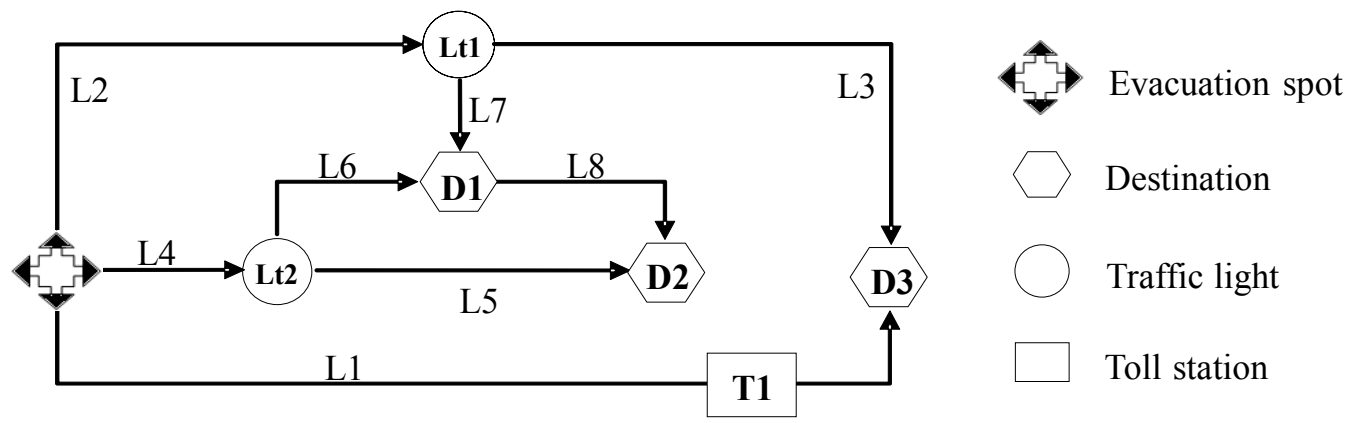

Figure 3. The traffic network of evacuation management system.

Table 1. The Modeling Parameters

\begin{tabular}{|c|c|c|c|c|c|c|c|}
\hline$L_{1}$ & $L_{2}$ & $L_{3}$ & $L_{4}$ & $L_{5}$ & $L_{6}$ & $L_{7}$ & $L_{8}$ \\
\hline 25 & 8 & 12 & 6 & 9 & 4 & 2.1 & 2.3 \\
\hline$V M_{1}$ & $V M_{2}$ & $V M_{3}$ & $V M_{4}$ & $V M_{5}$ & $V M_{6}$ & $V M_{7}$ & $V M_{8}$ \\
\hline 2250 & 700 & 1200 & 300 & 810 & 360 & 180 & 300 \\
\hline $\begin{array}{c}M X_{1} \\
{[1800,2000]}\end{array}$ & $\begin{array}{c}M X_{2} \\
{[450,505]}\end{array}$ & $\begin{array}{c}M X_{3} \\
{[990,1110]}\end{array}$ & $\begin{array}{c}M X_{4} \\
{[165,190]}\end{array}$ & $\begin{array}{c}M X_{5} \\
{[540,600]}\end{array}$ & $\begin{array}{c}M X_{6} \\
{[235,250]}\end{array}$ & $\begin{array}{c}M X_{7} \\
{[33,37]}\end{array}$ & $\begin{array}{c}M X_{8} \\
{[180,200]}\end{array}$ \\
\hline $\begin{array}{c}V_{1} \\
{[70,75]}\end{array}$ & $\begin{array}{c}V_{2} \\
{[27,30]}\end{array}$ & $\begin{array}{c}V_{3} \\
{[36,40]}\end{array}$ & $\begin{array}{c}V_{4} \\
{[20,25]}\end{array}$ & $\begin{array}{c}V_{5} \\
{[45,50]}\end{array}$ & $\begin{array}{c}V_{6} \\
{[30,35]}\end{array}$ & $\begin{array}{c}V_{7} \\
{[27,30]}\end{array}$ & $\begin{array}{c}V_{8} \\
{[36,40]}\end{array}$ \\
\hline $\begin{array}{c}\text { path11 } \\
\text { link }(4,6)\end{array}$ & $\begin{array}{c}\text { path12 } \\
\operatorname{Link}(2,7)\end{array}$ & $\begin{array}{c}\text { path21 } \\
\operatorname{Link}(4,5)\end{array}$ & $\begin{array}{c}\text { path22 } \\
\operatorname{Link}(4,6,8)\end{array}$ & $\begin{array}{c}\text { path } 23 \\
\operatorname{Link}(2,7,8)\end{array}$ & $\begin{array}{l}\text { path31 } \\
\text { Link(1) }\end{array}$ & $\begin{array}{c}\text { path32 } \\
\operatorname{Link}(2,3)\end{array}$ & \\
\hline $\begin{array}{c}L t_{1} \\
{[0.03,0.04]}\end{array}$ & $\begin{array}{c}L t_{2} \\
{[0.04,0.05]}\end{array}$ & $\begin{array}{c}T t_{1} \\
{[0.10,0.15]}\end{array}$ & $\begin{array}{c}T e_{1} \\
{[0.133,0.171]}\end{array}$ & $\begin{array}{c}\mathrm{Te}_{2} \\
{[0.111,0.143]}\end{array}$ & $\begin{array}{c}T e_{3} \\
{[0.089,0.114]}\end{array}$ & & \\
\hline $\begin{array}{l}N_{1} \\
130\end{array}$ & $\begin{array}{l}N_{2} \\
150\end{array}$ & $\begin{array}{l}N_{3} \\
120\end{array}$ & $\begin{array}{c}r_{1} \\
{[18,20]}\end{array}$ & $\begin{array}{c}r_{2} \\
{[27,30]}\end{array}$ & $\begin{array}{c}r_{3} \\
{[54,60]}\end{array}$ & & \\
\hline $\begin{array}{c}E I C_{1} \\
{[355,379]}\end{array}$ & $\begin{array}{c}E I C_{2} \\
{[603,619]}\end{array}$ & $\begin{array}{c}E I C_{3} \\
{[1183,1298]}\end{array}$ & $\begin{array}{c}\alpha_{1} \\
81.8760\end{array}$ & $\begin{array}{c}\alpha_{2} \\
138.905\end{array}$ & $\begin{array}{c}\alpha_{3} \\
454.813\end{array}$ & $\begin{array}{c}\beta_{1} \\
-1.8551\end{array}$ & $\begin{array}{c}\beta_{2} \\
-3.1472\end{array}$ \\
\hline $\begin{array}{c}\beta_{3} \\
-11.811\end{array}$ & $\begin{array}{c}\gamma_{1} \\
0.0123\end{array}$ & $\begin{array}{c}\gamma_{2} \\
0.0209\end{array}$ & $\begin{array}{c}\gamma_{3} \\
0.0878\end{array}$ & \multicolumn{2}{|c|}{$E p$} & & \\
\hline $\begin{array}{l}C_{11} \\
2.5 \\
\end{array}$ & $\begin{array}{c}C_{12} \\
4 \\
\end{array}$ & $\begin{array}{c}C_{13} \\
6 \\
\end{array}$ & $\begin{array}{c}F_{1} \\
{[1.10,1.40]}\end{array}$ & $\begin{array}{c}F_{2} \\
{[1.50,1.80]}\end{array}$ & $\begin{array}{c}F_{3} \\
{[2.40,2.80]}\end{array}$ & & \\
\hline $\begin{array}{c}C e_{1} \\
{[3.3,4.2]}\end{array}$ & $\begin{array}{c}C e_{2} \\
{[4.5,5.4]}\end{array}$ & $\begin{array}{c}C e_{3} \\
{[7.2,8.4]}\end{array}$ & $\begin{array}{c}B e_{1} \\
{[5.0,7.5]}\end{array}$ & $\begin{array}{c}\mathrm{Be}_{2} \\
{[8.0,11.0]}\end{array}$ & $\begin{array}{c}\mathrm{Be}_{3} \\
{[13.0,18.0]}\end{array}$ & $\begin{array}{c}G_{11} \\
1.75\end{array}$ & $\begin{array}{c}G_{12} \\
1.50\end{array}$ \\
\hline$G_{13}$ & $G_{21}$ & $G_{22}$ & $G_{23}$ & $G_{31}$ & $G_{32}$ & $G_{33}$ & \\
\hline 1.25 & 2.00 & 1.75 & 1.50 & 3.00 & 2.50 & 2.00 & \\
\hline
\end{tabular}

preferably used on time-saving paths. To reach destination 3 , most of the vehicles (i.e., $[52,96])$ would be allocated to path 31 , while only a small portion (i.e., 5) would be diverted to path 32. Large-sized vehicles account for most of those which would be directed to destination 3. Generally, under the three levels of evacuee numbers, large-sized vehicles would be preferred due to their lower transportation costs (per passenger) and $\mathrm{CO}$ emission rates (per passenger) compared with smaller ones. In contrast, small-sized vehicles would only be considered when the large- or medium-size ones are completely allocated. Under a lower level of evacuees, fewer small-sized vehicles would be allocated, and most of the solutions for the numbers of large-sized vehicles are intervals; comparatively, under a higher level of evacuees, small- and medium-sized vehicles are more likely to be adopted due to the shortage of large-sized ones, and the solutions for the amounts of large- 
sized vehicles are mostly deterministic.

Table 2. The Number of Evacuees to Each Destination and the Associated Probability

\begin{tabular}{cccc}
\hline$R_{s j}$ & $\begin{array}{c}\text { Level } \\
\text { description }\end{array}$ & $\begin{array}{c}\text { Number of evacuees } \\
\text { to each destination }\end{array}$ & $\begin{array}{c}\text { Probability } \\
\left(p_{s j}\right)\end{array}$ \\
\hline$R_{11}$ & Low & {$[2501,3000]$} & 0.31 \\
$R_{12}$ & Medium & {$[3001,3500]$} & 0.36 \\
$R_{13}$ & High & {$[3501,4000]$} & 0.33 \\
$R_{21}$ & Low & {$[3001,3500]$} & 0.26 \\
$R_{22}$ & Medium & {$[3501,4000]$} & 0.44 \\
$R_{23}$ & High & {$[4001,4500]$} & 0.30 \\
$R_{31}$ & Low & {$[2001,2500]$} & 0.30 \\
$R_{32}$ & Medium & {$[2501,3000]$} & 0.42 \\
$R_{33}$ & High & {$[3001,3500]$} & 0.28 \\
\hline
\end{tabular}

Table 3. The Number of Vehicles Pre-allocated to Each Route

\begin{tabular}{cccccc}
\hline$x_{1 a i}$ & $\begin{array}{c}\text { Number of } \\
\text { Vehicle }\end{array}$ & $x_{2 a i}$ & $\begin{array}{c}\text { Number of } \\
\text { Vehicle }\end{array}$ & $x_{3 a i}$ & $\begin{array}{c}\text { Number of } \\
\text { Vehicle }\end{array}$ \\
\hline$x_{111}$ & {$[0,42]$} & $x_{211}$ & {$[0,5]$} & $x_{311}$ & {$[1,2]$} \\
$x_{112}$ & {$[1,1]$} & $x_{212}$ & {$[0,25]$} & $x_{312}$ & {$[0,20]$} \\
$x_{113}$ & {$[5,8]$} & $x_{213}$ & {$[0,19]$} & $x_{313}$ & {$[35,45]$} \\
$x_{121}$ & {$[0,30]$} & $x_{221}$ & 5 & $x_{321}$ & {$[0,2]$} \\
$x_{122}$ & {$[45,55]$} & $x_{222}$ & {$[2,5]$} & $x_{322}$ & {$[0,5]$} \\
$x_{123}$ & {$[10,15]$} & $x_{223}$ & {$[20,60]$} & $x_{323}$ & {$[5,25]$} \\
$/$ & $/$ & $x_{231}$ & {$[0,11]$} & $/$ & $/$ \\
$/$ & $/$ & $x_{232}$ & {$[0,25]$} & $/$ & $/$ \\
$/$ & $/$ & $x_{233}$ & {$[2,5]$} & $/$ & $/$ \\
\hline
\end{tabular}

Table 4 also indicates that, the solution for the objective function value provides two extremes of the minimum time $\left(t_{\text {opt }}^{ \pm}=[3556,6181]\right)$ which are required to evacuate all of the event-participants to their destinations. As the actual value of each variable or parameter vary within its two bounds, the total evacuation time may change correspondingly between $t_{\text {opt }}^{-}$ and $t_{\text {opt }}^{+}$under a variety of reliability levels. Planning for the lower bound of the objective function value would lead to a lower evacuation time and emission level, but a higher risk of violating the evacuation demand constraints. In contrast, planning for the upper bound of the objective junction value would result in higher system stability; but this would also lead to higher system cost and emission level. Therefore, these results could help analyze the tradeoff between system efficiency and reliability. Desired decision alternatives can thus be generated from the solutions according to projected applicable system conditions.

\section{Conclusions}

A two-stage interval-stochastic evacuation management
(TISEM) model was developed for supporting environmentoriented evacuation management under uncertainty. Through TISEM, minimized evacuation time could be achieved with a comprehensive consideration of economic cost and environmental target. Interval solutions could be obtained from the model, which were useful for facilitating the generation of decision alternatives under various system conditions. In addition, TISEM was helpful for dynamic analysis of vehicle assignment within a multi-stage and multi-level context.

A study case was used to demonstrate the applicability of TISEM. Useful solutions were obtained. The results revealed that, large-sized vehicles would be preferably adopted due to their lower transportation costs (per passenger) and $\mathrm{CO}$ emissions (per passenger) compared to small- and medium sized ones. In contrast, small-sized vehicles would only be adopted when large- or medium-sized ones were completely allocated. Under a lower level of evacuees, fewer small-sized vehicles would be allocated for evacuation, and most of the solutions for the numbers of large-size vehicles were intervals; comparatively, under a higher level of evacuees, small- and mediumsized vehicles were more likely to be adopted due to the exhaustion of large-sized ones, and the solutions for the amounts of large-size vehicles were mostly deterministic.

This study made the first attempt to plan evacuation management systems through a two-stage interval-stochastic programming (TISP) approach. With the integration of two-stage stochastic programming (TSP) and interval-parameter programming (IPP) methods, TISEM improves upon the conventional evacuation management models by directly communicating uncertain information into optimization efforts. The study results have demonstrated that TISEM is applicable to real-world evacuation management problems that are associated with extensive uncertainties within a multi-stage context.

Acknowledgments. This research was supported by the Major State Basic Research Development Program of MOST (2005CB724200 and 2006CB403307) and the Natural Science and Engineering Research Council of Canada.

\section{References}

Bakuli, D.L. and Smith, J.M. (1996). Resource allocation in statedependent emergency evacuation networks, European Journal of Operational Research, 89, 543-555, doi:10.1016/0377-2217(94)00 $230-4$.

Cova, T.J. and Johnson, J.P. (2003). A network flow model for lanebased evacuation routing, Transp. Res. A, 37, 579-604, doi:10.101 6/S0965-8564(03)00007-7.

Decker, S.H., Varano, S.P. and Greene, J.R. (2007). Routine crime in exceptional times: The impact of the 2002 Winter Olympics on citizen demand for police services, J. Crim. Just., 35(1), 89-101, doi: 10.1016/j.jcrimjus.2006.11.018.

Ettema, D. and Timmermans, H. (2006). Costs of travel time uncertainty and benefits of travel time information: Conceptual model and numerical examples, Transp. Res. C, 14, 335-350, doi:10.1016 j.trc.2006.09.001

Frantzeskakis, J.M. and Frantzeskakis, M.J. (2006). Athens 2004 Olympic Games: Transportation Planning, Simulation and Traffic Management, ITE Journal, 76(10), 26-33. 
Table 4. Solutions of the Study Case

\begin{tabular}{|c|c|c|c|c|c|c|c|c|c|}
\hline \multirow{2}{*}{$x_{s a i}$} & \multirow{2}{*}{$\Delta x_{s a i}$} & \multirow{2}{*}{$y_{\text {sai opt }}$} & \multirow{2}{*}{$x_{\text {sai opt }}^{ \pm}$} & \multicolumn{3}{|c|}{$D_{\text {saij opt }}^{ \pm}$} & \multicolumn{3}{|c|}{$A_{\text {saij opt }}^{ \pm}$} \\
\hline & & & & $j=1$ & $j=2$ & $j=3$ & $j=1$ & $j=2$ & $j=3$ \\
\hline$x_{111}$ & 42 & 0 & 0 & 0 & {$[0,1]$} & {$[0,1]$} & 0 & {$[0,1]$} & {$[0,1]$} \\
\hline$x_{112}$ & 0 & 1 & 1 & 0 & {$[0,20]$} & {$[0,28]$} & 1 & {$[1,21]$} & {$[1,29]$} \\
\hline$x_{113}$ & 3 & 0 & 5 & 0 & 0 & 0 & 5 & 5 & 5 \\
\hline$x_{121}$ & 30 & 0 & 0 & 0 & 1 & 2 & 0 & 1 & 2 \\
\hline$x_{122}$ & 10 & 0.74 & 52 & 0 & {$[0,1]$} & {$[0,3]$} & 52 & {$[52,53]$} & {$[52,55]$} \\
\hline$x_{123}$ & 5 & 0 & 10 & {$[0,14]$} & {$[8,12]$} & 16 & {$[10,24]$} & {$[18,22]$} & 26 \\
\hline$x_{211}$ & 5 & 0 & 0 & 0 & {$[0,1]$} & {$[0,17]$} & 0 & {$[0,1]$} & {$[0,17]$} \\
\hline$x_{212}$ & 25 & 0.05 & 1 & 0 & 0 & {$[0,15]$} & 1 & 1 & {$[1,16]$} \\
\hline$x_{213}$ & 19 & 0 & 0 & 0 & 0 & 0 & 0 & 0 & 0 \\
\hline$x_{221}$ & 0 & 1 & 5 & 0 & 0 & 0 & 5 & 5 & 5 \\
\hline$x_{222}$ & 3 & 1 & 5 & 0 & 0 & {$[0,7]$} & 5 & 5 & {$[5,12]$} \\
\hline$x_{223}$ & 40 & 0.1 & 24 & 0 & 0 & 0 & 24 & 24 & 24 \\
\hline$x_{231}$ & 11 & 1 & 11 & {$[0,1]$} & 1 & 2 & {$[11,12]$} & 12 & 13 \\
\hline$x_{232}$ & 25 & 1 & 25 & {$[0,1]$} & {$[0,1]$} & 0 & {$[25,26]$} & {$[25,26]$} & 25 \\
\hline$x_{233}$ & 3 & 1 & 5 & {$[0,14]$} & {$[8,23]$} & 16 & {$[5,19]$} & {$[13,28]$} & 21 \\
\hline$x_{311}$ & 1 & 0 & 1 & 0 & 1 & {$[2,45]$} & 1 & 2 & {$[3,46]$} \\
\hline$x_{312}$ & 20 & 0 & 0 & 0 & {$[0,28]$} & {$[10,11]$} & 0 & {$[0,28]$} & {$[10,11]$} \\
\hline$x_{313}$ & 10 & 0 & 35 & {$[0,6]$} & 1 & 4 & {$[35,41]$} & 36 & 39 \\
\hline$x_{321}$ & 2 & 0 & 0 & 0 & 0 & 0 & 0 & 0 & 0 \\
\hline$x_{322}$ & 5 & 0 & 0 & 0 & 0 & 0 & 0 & 0 & 0 \\
\hline$x_{323}$ & 20 & 0 & 5 & 0 & 0 & 0 & 5 & 5 & 5 \\
\hline$t_{o p t}^{ \pm}$ & & & & & {$[3556,61$} & & & & \\
\hline
\end{tabular}

Graata, E., Middena C. (1999). Complex evacuation: effects of motivation level and slope of stairs on emergency egress time in a sports stadium, Safety Science, 31, 127-141, doi:10.1016/S0925-7 535(98)00061-7.

Grivas, G. and Chaloulakou, A. (2006). Artificial neural network models for prediction of PM10 hourly concentrations, in the Greater Area of Athens, Greece, Atmospheric Environment, 40, 1216-1229, doi:10.1016/j.atmosenv.2005.10.036.

Hobeika, A.G. and Kim, C. (1998). Comparison of Traffic Assignments in Evacuation Modeling, IEEE Transactions on Engineering Management, 45(2), 192-198.

Huang, G. and Moore, R.D. (1993). Grey Linear Programming, its Solving Approach, and its Application, International Journal of Systems Science, 24, 159-172.

Huang, G., Baetz, B.W. and Patry, G.G. (1994). Capacity Planning for Municipal Solid Waste Management Systems under Uncertainty: A Grey Fuzzy Dynamic Programming (GFDP) Approach, J. Urban Plann. Dev., 120, 132-156.

Huang, G.H. (1996). IPWM: An Interval Parameter Water Quality Management Model, Eng. Optimiz., 26, 79-103.

Huang, G.H. and Louck, D.P. (2000). An Inexact Two-Stage Stochastic Programming Model for Water Resources Management under Uncertainty, Civ. Eng. Environ. Syst., 17, 95-118, doi:10.1007/s11 269-007-9206-8.

Li, Y.P. and Huang, G.H. (2006). An inexact two-stage mixed integer linear programming method for solid waste management in the City of Regina, J. Environ. Manage., 81, 188-209, doi:10.1016/j.je nvman.2005.10.007.
Lv, W., Wang, Y., Querol, X., Zhuang, X., Alastuey, A., López, A. and Viana, M. (2006). Geochemical and statistical analysis of trace metals in atmospheric particulates in Wuhan, central China, Environ. Geol., 51, 121-132, doi:10.1007/s00254-006-0310-5.

Maqsood, I. and Huang, G.H. (2003). A two-stage interval-stochastic programming model for waste management under uncertainty, Journal of the Air and Waste Management Association, 53(5), 540552.

Maqsood, I., Huang, G.H. and Zeng, G.M. (2004). An inexact twostage mixed integer linear programming model for waste management under uncertainty, Civ. Eng. Environ. Syst., 21(3), 187-206.

Querol, X., Zhuang X., Alastuey A., Viana M., Lv W., Wang Y., López A., Zhu Z., Wei H. and Xu S. (2006). Speciation and sources of atmospheric aerosols in a highly industrialized emerging mega-city in Central China, J. Environ. Monit., 8, 1049-1059.

Sattayhatewa, P. and Ran, B. (2000). Developing a dynamic traffic management model for nuclear power plant evacuation, Transportation Research Board Annual Meeting, 1-24.

Southworth, F. (1991). Regional evacuation modeling: a state-of-theart review, Oak Ridge National Labs, ORNL/TM-11740.

Suleyman, T. (1995). An integrated emergency management decision support system for hurricane emergencies, Safety Science, 20, 3948, doi:10.1016/0925-7535(94)00065-B.

Tan, Q., Huang, G.H. and Wu, C.Z. (2008). Development of an Inexact Fuzzy Robust Programming Model for Integrated Evacuation Management under Uncertainty, in press.

Urbanik, T. (2000). Evacuation time estimates for nuclear power plants, J. Hazard. Mater., 75, 165-180, doi:10.1016/S0304-3894(0 
0)00178-3.

Waller, S.T., Schofer, J.L. and Ziliaskopoulos, A.K. (2001). Evaluation with traffic assignment under demand uncertainty, J. Trans. Res. Board, 1771, 69-75, doi:10.3141/1771-09.

Waller, S.T. and Ziliaskopoulos, A.K. (2006). A chance-constrained based stochastic dynamic traffic assignment model: Analysis, formulation and solution algorithms, Transp. Res. C, 14, 418- 427, doi:10.1016/j.trc.2006.11.002.
Wu, C.Z., Huang, G.H., Yan, X.P., Cai, Y.P. and Li, Y.P. (2008). An inexact optimization model for evacuation planning. Kybernetes, In press.

Yi, W. and Zdamar, L.O. (2007). A dynamic logistics coordination model for evacuation and support in disaster response activities, European Journal of Operational Research, 179, 1177-1193, doi:10. 1016/j.ejor.2005.03.077. 